\title{
CALIBRAÇÃO EM SERVIÇO DE CÂMARA DIGITAL DE BAIXO CUSTO COM O USO DE PONTOS DE APOIO ALTIMÉTRICO
}

\author{
On-the-Job Calibration of low cost digital camera with vertical control points \\ PAULA DEBIASI ${ }^{1,2}$ \\ FABIANO HAINOSZ 1 \\ EDSON A. MITISHITA ${ }^{1}$ \\ ${ }^{1,2}$ Universidade Federal Rural do Rio de Janeiro \\ Instituto de Tecnologia / Departamento de Engenharia \\ Rodovia BR 465, $\mathrm{Km} 7$, \\ CEP- 23.890-000 - Seropédica - Rio de Janeiro - Brasil \\ paula@ufrrj.br; \\ ${ }^{1}$ Universidade Federal do Paraná \\ Programa de Pós-Graduação em Ciências Geodésicas \\ Centro Politécnico - Jardim das Américas - Caixa Postal 19001 \\ CEP: 81.531-990 - Curitiba - PR - BR \\ fabiano.h@lactec.org.br, mitishita@ufpr.br
}

\begin{abstract}
RESUMO
O conhecimento dos parâmetros que definem a geometria interna de uma câmara é fundamental em trabalhos fotogramétricos. Porém, é de suma importância que o processo de calibração da câmara seja realizado em condições técnicas e ambientais similares ao aerolevantamento. No georreferenciamento direto os parâmetros de orientação exterior da câmara podem ser determinados com base nas observações GPS e INS. A utilização destes parâmetros, em um processo de calibração com parâmetros adicionais, possibilita a atenuação de correlações entre parâmetros de orientação interior e exterior. Este artigo investiga o uso dos parâmetros de posição da câmara e diferentes configurações de pontos de apoio altimétrico na calibração em serviço de uma câmara digital Kodak DSC Pro SLR/c. O impacto do uso de faixas cruzadas na calibração em serviço foi avaliado pela análise da correlação estatística entre os parâmetros de orientação interior e exterior. Finalmente, as exatidões planialtimétricas obtidas nos experimentos de calibração em serviço, com diferentes configurações de pontos de apoio altimétrico e faixas de voo, foram
\end{abstract}


comparadas através da análise das discrepâncias de pontos de verificação.

Palavras-chave: Calibração em Serviço; Câmara de Baixo Custo; Campo de Prova.

\begin{abstract}
Knowledge of the internal camera geometry is an important task to perform photogrammetric works. However, IOPs from camera calibration using dataset with the same techniques and environmental conditions of the aerial survey is a prerequisite to produce photogrammetric mapping using direct georeferencing technique. In direct georeferencing, the exterior orientation parameters are determined based on GPS and INS observations. The use of these parameters, in the calibration process with additional ones, reduces the correlations between interior and exterior orientation parameters. This paper investigates the use of camera position and different configurations of vertical control points in the on-the-job calibration of digital camera Kodak DSC Pro SLR/c. The advantages of using cross strips to perform on-the job calibration were analyzed by statistical correlations between interior and exterior orientation parameters. Finally, the vertical and horizontal accuracies from the calibration experiments, with different configurations of vertical control points and flights strips were compared using the analysis of discrepancies in the check points.
\end{abstract}

Keywords: On-the-Job Calibration; Low Cost Camera; Ttest Field.

\title{
1. INTRODUÇÃO
}

A calibração dos sensores é de suma importância para o controle geométrico das imagens adquiridas. A utilização de um modelo adequado à geometria interna da câmara garante que as medições sobre as imagens, em teoria, estarão livres de erros sistemáticos, ou pelo menos que eles sejam insignificantes (LERMA e CABRELLES, 2007). A calibração de câmaras tem o propósito de estimar os parâmetros de orientação interior (POI) da câmara. Os POI compreendem a distância focal da câmara (f), as coordenadas do ponto principal $\left(\mathrm{x}_{0} \quad \mathrm{e} \mathrm{y}_{0}\right)$ e parâmetros que realizam as correções nas fotocoordenadas para compensar os desvios sofridos por admitir-se uma geometria perspectiva (HABIB, PULLIVELLI e MORGAN, 2004). A geometria perspectiva pode ser estabelecida pela condição de colinearidade, que rege que o ponto no espaço imagem, o centro de projeção da câmara $(\mathrm{CP})$ e o respectivo ponto no espaço objeto são colineares.

No caso da calibração geométrica de câmaras, diferentes métodos podem ser considerados: Métodos de Laboratório e Métodos de Campo (GALO E TOMMASELLI, 2011). Na calibração de câmaras realizada em laboratório a determinação dos parâmetros não reflete as condições de voo como os efeitos de temperatura, umidade e pressão de ar (YASTIKLI, TOTH e BRZEZINSKA, 2007; HONKAVAARA, 2008). Os métodos de campo oferecem soluções mais completas, pois permitem a determinação simultânea de todos os parâmetros de orientação interior num único ajustamento, realizado com abundância de observações e 
controle estatístico rigoroso do processo (ANDRADE, 2003). Neste caso, os POI são introduzidos como parâmetros adicionais às equações de colinearidade, ou seja, são tratados como incógnitas no ajustamento por mínimos quadrados. Tradicionalmente, na calibração de uma câmara, o método de calibração em campo utiliza um conjunto de fotografias convergentes tomadas de um campo de prova com um grande número de pontos de apoio (YASTIKLI, TOTH e BRZEZINSKA, 2007; HONKAVAARA et al., 2008).

Conforme Merchant (1980) quando as fotografias aéreas são verticais e tomadas em uma região plana, num processo de calibração de campo, onde os parâmetros de orientação exterior (POE) das imagens são recuperados simultaneamente aos $\mathrm{POI}$, os parâmetros de posição da câmara $\left(\mathrm{X}_{0}, \mathrm{Y}_{0}\right.$ e $\left.\mathrm{Z}_{0}\right)$ são diretamente correlacionados com as coordenadas do ponto principal e a distância focal, respectivamente $\left(\mathrm{x}_{0}, \mathrm{y}_{0} \mathrm{e} \mathrm{f}\right)$. Em outras palavras é impossível determinar-se simultaneamente os três pares de parâmetros quando a altura de voo é constante e o terreno é plano. Estas correlações podem ser atenuadas com a determinação das coordenadas do CP da câmara no aerolevantamento (YASTIKLI e JACOBSEN, 2005).

A crescente utilização de câmaras digitais de baixo custo, integradas com sistemas LIDAR (Light Detection And Rangig), para a produção de ortoimagens, motivou a realização de pesquisas que visam o estabelecimento de técnicas econômicas e eficientes de calibração de câmaras com base em recobrimentos aéreos (laser scanner e aerofotogramétrico realizados simultaneamente). Utiliza-se a terminologia "câmaras de baixo custo" para expressar câmaras digitais que não foram originalmente designadas para propósitos fotogramétricos. O LIDAR aerotransportado está fundamentado na integração do Laser (Light Amplification by Stimulated Emission of Radiation) scanner, GPS (Global Position System) e INS (Inertial Navigation System) para a determinação autônoma de coordenadas tridimensionais de pontos no terreno.

Considerando a possibilidade da utilização de um levantamento laser scanner no processo de calibração, esse trabalho tem por objetivo principal avaliar a viabilidade de realizar uma calibração em serviço de uma câmara digital de baixo custo empregando somente pontos de apoio altimétrico com diferentes configurações de pontos de apoio altimétrico e de faixas de voo. Com a integração da câmara com o sistema LIDAR foi possível a determinação da posição do CP da câmara no instante de tomada da imagem e a utilização dessa informação no processamento da calibração em serviço. Esse procedimento viabilizou a atenuação de correlações entre parâmetros de orientação exterior e interior e a minimização de pontos de apoio na calibração em serviço.

\section{CALIBRAÇÃO DE CÂMARAS}

Brown, em 1956, provou que é possível determinar os parâmetros de calibração das lentes simultaneamente com a determinação das coordenadas tridimensionais de pontos por um processo de fototriangulação por feixes de raios 
(CLARKE; FRYER, 1998). Esta técnica, quando não utiliza nenhuma informação externa de pontos de apoio, mas somente um conjunto de imagens convergentes tomadas com diferentes pontos de vista, sendo pelo menos uma rotacionada de $90^{\circ}$ em relação às demais, é denominada de autocalibração. A definição arbitrária da posição e orientação de uma imagem define o referencial necessário para a determinação dos POE das outras imagens, posições tridimensionais de pontos medidos e POI envolvidos no processo. Apesar da não utilização de pontos de apoio com suficiente variação altimétrica, o método de calibração é robusto, pois juntamente com o conjunto de imagens convergentes, a utilização de pelo menos uma imagem com um giro de $90^{\circ}$ em torno do eixo óptico da câmara quebra a dependência linear entre os parâmetros de orientação interior e exterior e habilita a separação dos coeficientes da distorção radial simétrica com os da distorção descentrada (ANDRADE, 2003).

Segundo Clarke e Fryer (1998) o termo calibração em serviço é muitas vezes confundido com autocalibração. Entretanto, na calibração em serviço um conjunto de pontos de apoio é empregado e o processo pode ser considerado como sendo uma fototriangulação por feixes de raios com parâmetros adicionais. No caso de fotografias aéreas, procedimentos adicionais são empregados para quebrar a dependência linear entre parâmetros de orientação exterior e interior, tais como: tomadas de fotos inclinadas, utilização de faixas com diferentes alturas de voo, conjunto de pontos de apoio com grande variação nas coordenadas altimétricas e fixação da posição das estações de exposição (centro de projeção). Maiores detalhes de calibrações em serviço realizadas com recobrimentos aéreos podem ser vistos em Bazan et al. (2007); Ruy et al. (2008); Mitishita et al. (2010); Côrtes (2010).

Brown (1971) desenvolveu o método plumb-line de calibração de câmaras utilizando feições retas. Lerma e Cabrelles (2007) determinaram os parâmetros de distorção radial e descentrada de uma câmara digital não-métrica usando o método de calibração plumb-line. Habib, Morgan e Lee (2002) incorporaram feições retas no ajustamento por feixes perspectivos com autocalibração de imagens de quadro. Esses autores acreditam que as feições retas podem impactar os processos de calibração de câmaras devido às deformações e distorções serem mapeadas ao longo de suas direções como funções contínuas. Habib e Morgan (2003) compararam a eficiência da calibração em serviço utilizando pontos como controle e incorporando linhas retas no modelo matemático de calibração. Os autores demonstram que o cálculo de coordenadas do espaço objeto utilizando os POI advindos dos dois processos de calibração em serviço (somente com pontos e com linhas retas e pontos) são similares. Porém, eles alegam que a construção de um campo de prova com feições retas é mais fácil de ser estabelecida, além dos experimentos de calibração com linhas retas não apresentarem correlações entre parâmetros de orientação exterior e interior verificadas nos experimentos utilizando pontos. Habib, Pullivelli e Morgan (2004) também propõem um campo de prova com feições retas e pontuais como controle para a calibração em serviço. 
Honkavaara (2008) demonstra uma metodologia de calibração de câmaras fotogramétricas digitais utilizando um campo de prova para a calibração geométrica, radiométrica e da resolução espacial simultaneamente. Em Remondino e Fraser (2006) são discutidos os principais aspectos da autocalibração na Fotogrametria a curta distância e na visão computacional. Cronk, Fraser e Hanley (2006) descrevem um novo método de calibrar automaticamente câmaras digitais coloridas utilizando a autocalibração. Os atributos de cor RGB (red, green e blue) são explorados para detectar e identificar automaticamente alvos pré-sinalizados. $\mathrm{O}$ impacto da aberração cromática é discutido, pois diferentes valores de distância focal e coeficientes de distorção radial são encontrados para cada banda R, G e B.

\subsection{Estabilidade da câmara e o georreferenciamento direto}

Câmaras digitais de baixo custo não são construídas para a reconstrução fotogramétrica, e não possuem a estabilidade no mapeamento como as câmaras fotogramétricas. A sua estabilidade requer uma análise mais profunda, devendo-se avaliar se as características internas desta câmara permanecem estáveis durante o tempo (HABIB et al., 2008). Ainda segundo os mesmos autores, se a câmara é estável os POI não variam durante o tempo.

Em Mitishita et al. (2009) foi verificada a estabilidade geométrica de uma câmara de baixo custo com a variação de temperatura. Nos experimentos de calibração foram utilizados dois campos de prova, sendo um bidimensional e outro tridimensional. Duas calibrações foram realizadas submetendo a câmara a temperaturas próximas a $0^{\circ} \mathrm{C}$ e quatro calibrações com a temperatura próxima a $25^{\circ} \mathrm{C}$. Os valores de POI das seis calibrações não foram similares e a câmara foi considerada geometricamente instável. No entanto, os resultados não provaram que a instabilidade geométrica da câmara é decorrente da variação da temperatura.

Mitishita et al. (2010) calibrou uma câmara digital de baixo custo em duas diferentes situações: calibração independente e calibração em serviço. Na calibração em serviço foram fixadas as posições de tomada das imagens no ajustamento para minimizar as correlações entre os POI e POE. Os parâmetros de distorção das lentes foram similares nas duas calibrações, e as correlações entre parâmetros foram minimizadas na calibração em serviço.

Processos fotogramétricos utilizando POE advindos do georreferenciamento direto possuem ligação direta com a estabilidade da câmara (HABIB et al., 2006). Os vetores de distância entre a câmara e o INS podem ser medidos diretamente por técnicas geodésicas e/ou topográficas, mas as diferenças angulares entre a câmara e o INS não podem ser determinadas diretamente. As diferenças angulares entre o INS e a câmara só podem ser determinadas pela comparação da solução fotogramétrica (Aerotriangulação convencional) com resultados obtidos no préprocessamento GPS/INS ou considerando estas diferenças angulares como incógnitas no processo de fototriangulação por feixes de raios (WEGMANN, HEIPKE e JACOBSEN, 2004; YASTIKLI e JACOBSEN, 2005). Segundo Wegmann; Heipke e Jacobsen (2004) o pré-processamento GPS/INS consiste em 
transformar os dados brutos dos sistemas GPS e INS em trajetórias do CP da câmara no espaço objeto e determinar valores de orientação desta trajetória (yaw, pitch e roll).

Pequenas mudanças nos POI produzem efeitos na qualidade da reconstrução do espaço objeto. Em compensação no georreferenciamento indireto, ou seja, na determinação dos POE por aerotriangulação de um bloco de imagens, problemas na acurácia dos POI podem ser compensados no cálculo dos POE. Experimentos realizados por Mitishita et al. (2009) mostraram que a câmara digital de baixo custo analisada foi considerada estável em experimentos com georreferenciamento indireto. Porém esta câmara foi considerada instável no georreferenciamento direto, reafirmando a necessidade de acurados POI.

\section{EXPERIMENTOS DE CALIBRAÇÃO EM SERVIÇO}

Nos experimentos de calibração em serviço foram utilizadas fotografias aéreas advindas de uma câmara digital de baixo custo da Kodak modelo DCS Pro SLR/c. A dimensão do CCD da câmara é de 4500x3000 pixels e o tamanho do pixel de $8 \mu \mathrm{m}$. O bloco fotogramétrico contém 31 fotografias adquiridas com altura de voo de $1.000 \mathrm{~m}$. As fotografias foram tomadas no dia 30/05/2011 em um campo de prova, implantado pelos autores, na cidade de Ponta Grossa - PR. O campo de prova possui 33 pontos pré-sinalizados em formato de círculos com diâmetro de $0,60 \mathrm{~m}$. A forma e a dimensão dos alvos implantados foram determinadas a fim de prover a mais precisa visualização e medição destes nas fotografias. O diâmetro dos alvos foi calculado em função do GSD (Ground Sample distance - tamanho do pixel no terreno) das fotografias que é de $0,225 \mathrm{~m}$, de forma que o alvo tivesse dimensão de cerca de 3 vezes o GSD. A figura 1 mostra a distribuição das quatro faixas de voo e dos alvos pré-sinalizados.

Figura 1 - Configuração do bloco fotogramétrico dos experimentos de calibração em serviço e a distribuição dos pontos pré-sinalizados.

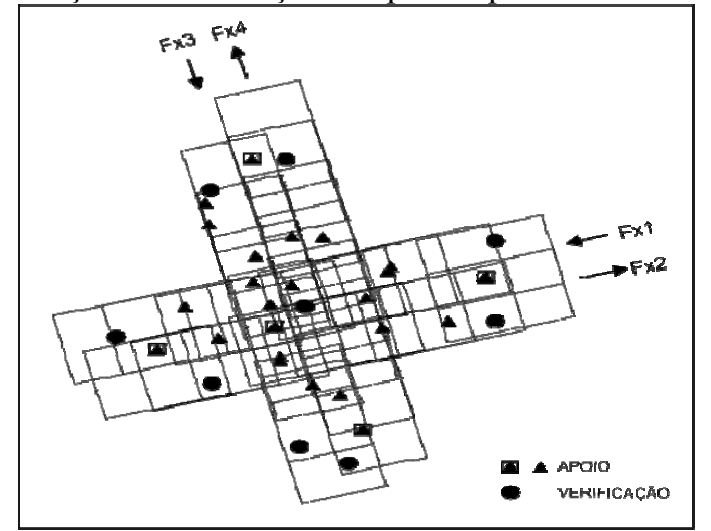

Bol. Ciênc. Geod., sec. Artigos, Curitiba, v. 18, no 2, p.225-241, abr-jun, 2012. 
As coordenadas dos pontos pré-sinalizados foram obtidas por receptor GPS no método de posicionamento estático com precisão de $0,03 \mathrm{~m}$. A diferença máxima de altitude entre os pontos pré-sinalizados é de aproximadamente 57 metros, que corresponde a cerca de $5 \%$ da altura de voo. Apesar da variação do terreno não ser significativa (inferior a $20 \%$ da altura de voo), utiliza-se nos experimentos injunções nos $\mathrm{CP}$, que reduzem a correlação entre os POI e POE.

Inicialmente, foram realizados experimentos de calibração em serviço somente com as faixas 1 e 2 apresentadas na figura 1. A configuração deste bloco teve como intuito se aproximar à condição de um recobrimento aerofotogramétrico convencional, ou seja, sem o uso de faixas cruzadas. Em todos os experimentos, a posição do $\mathrm{CP}$ da câmara $\left(\mathrm{X}_{0}, \mathrm{Y}_{0}\right.$ e $\left.\mathrm{Z}_{0}\right)$ foi utilizada como injunção no ajustamento, com precisão de $10 \mathrm{~cm}$. Essas coordenadas foram determinadas pela integração direta da câmara com o sistema LIDAR. Maiores detalhes podem ser obtidos em Martins (2010). A Tabela 1 apresenta as configurações dos pontos de apoio utilizadas nos quatro experimentos.

Tabela 1 - Experimentos de calibração em serviço com duas faixas

\begin{tabular}{ccc}
\hline Experimento & Configuração do Bloco & Parâmetros Injuncionados \\
\hline 1 & Faixas 1,2 & 15 Pontos de Apoio Planialtimétrico \\
2 & Faixas 1, 2 & 15 Pontos de Apoio Altimétrico \\
3 & Faixas 1, 2 & 3 Pontos de Apoio Altimétrico \\
4 & Faixas 1,2 & 1 Ponto de Apoio Altimétrico \\
\hline
\end{tabular}

No experimento 1 foram utilizados 15 pontos de apoio planialtimétrico distribuídos homogeneamente nas faixas 1 e 2 (pontos representados como triângulos e quadrados na figura 1). Como o propósito desta pesquisa foi de avaliar o uso de pontos altimétricos na calibração em serviço, este experimento foi realizado para servir de referência aos demais. No experimento 2 utilizou-se 15 pontos de apoio altimétrico esparsamente distribuídos nas duas faixas de voo (pontos simbolizados como triângulos e quadrados na figura 1). No experimento 3 utilizou-se 3 pontos de apoio altimétrico (pontos sinalizados com um quadrado nas faixas 1 e 2 da figura 1). Esses pontos foram localizados nas duas extremidades e no centro do bloco, de forma a propiciar uma configuração geométrica adequada de pontos de apoio altimétrico no bloco. No experimento 4 utilizou-se apenas de um ponto de apoio altimétrico localizado no centro do bloco (ponto sinalizado com um quadrado na figura 1). O objetivo destes dois últimos experimentos foi de verificar a viabilidade da calibração em serviço com o menor número possível de pontos de apoio altimétrico, visto que neste trabalho utilizou-se também da posição do CP da câmara nos processamentos de calibração.

Em todos os experimentos de calibração em serviço foi empregado como desvio padrão das fotocoordenadas $1 / 2$ pixel $(0,004 \mathrm{~mm})$, como desvio padrão das coordenadas de campo $3 \mathrm{~cm}$ e como desvio padrão das coordenadas do $\mathrm{CP} 10 \mathrm{~cm}$. A 
tabela 2 contém os valores dos POI significativos na análise da matriz variância e covariância do ajustamento, os seus desvios padrão, o erro médio quadrático (EMQ) dos resíduos das coordenadas dos pontos de apoio e centros de projeção e o EMQ dos resíduos das fotocoordenadas resultantes em cada um dos experimentos de calibração em serviço realizado.

Tabela 2 - POI, EMQ das coordenadas dos Pontos de Apoio e EMQ das fotocoordenadas nos experimentos de calibração em serviço.

\begin{tabular}{|c|c|c|c|c|}
\hline \multicolumn{5}{|c|}{ Parâmetros de Orientação Interior } \\
\hline & 1 & 2 & 3 & 4 \\
\hline $\mathrm{f}(\mathrm{mm})$ & 34,248 & 34,238 & 34,238 & 34,236 \\
\hline$\sigma_{\mathrm{f}}(\mathrm{mm})$ & 0,002 & 0,003 & 0,004 & 0,005 \\
\hline $\mathrm{x}_{0}(\mathrm{~mm})$ & 0,067 & 0,069 & 0,069 & 0,068 \\
\hline$\sigma_{\mathrm{x} 0}(\mathrm{~mm})$ & 0,002 & 0,002 & 0,002 & 0,002 \\
\hline $\mathrm{y}_{0}(\mathrm{~mm})$ & 0,264 & 0,263 & 0,264 & 0,267 \\
\hline$\sigma_{\mathrm{y} 0}(\mathrm{~mm})$ & 0,004 & 0,005 & 0,006 & 0,006 \\
\hline $\mathrm{k}_{1}\left(\mathrm{~mm}^{-2}\right)$ & $-9,099 \mathrm{E}-05$ & $-9,117 \mathrm{E}-05$ & $-9,094 \mathrm{E}-05$ & $-9,097 \mathrm{E}-05$ \\
\hline$\sigma_{\mathrm{k} 1}\left(\mathrm{~mm}^{-2}\right)$ & $5,525 \mathrm{E}-07$ & $5,509 \mathrm{E}-07$ & 5,693E-07 & $5,476 \mathrm{E}-07$ \\
\hline $\mathrm{k}_{2}\left(\mathrm{~mm}^{-4}\right)$ & $1,042 \mathrm{E}-07$ & $1,042 \mathrm{E}-07$ & $1,034 \mathrm{E}-07$ & $1,036 \mathrm{E}-07$ \\
\hline$\sigma_{\mathrm{k} 2}\left(\mathrm{~mm}^{-4}\right)$ & $1,286 \mathrm{E}-09$ & 1,293E-09 & $1,351 \mathrm{E}-09$ & 1,299E-09 \\
\hline \multicolumn{5}{|c|}{ EMQ dos resíduos das coordenadas dos pontos de apoio e CPs } \\
\hline EMQ X (m) & 0,002 & - & - & - \\
\hline EMQ Y (m) & 0,006 & - & - & - \\
\hline EMQ Z (m) & 0,002 & 0,003 & 0,004 & 0,000 \\
\hline $\operatorname{EMQ~X}_{0}(\mathrm{~m})$ & 0,037 & 0,030 & 0,027 & 0,026 \\
\hline $\mathrm{EMQ} \mathrm{Y}_{0}(\mathrm{~m})$ & 0,026 & 0,026 & 0,026 & 0,025 \\
\hline $\mathrm{EMQ} \mathrm{Z}_{0}(\mathrm{~m})$ & 0,070 & 0,055 & 0,057 & 0,050 \\
\hline \multicolumn{5}{|c|}{ EMQ dos resíduos das fotocoordenadas } \\
\hline EMQ x (mm) & 0,001 & 0,001 & 0,001 & 0,001 \\
\hline EMQ y (mm) & 0,002 & 0,002 & 0,002 & 0,002 \\
\hline
\end{tabular}

Analisando a tabela 2, verifica-se que os POI determinados nos experimentos com as configurações propostas de pontos de apoio altimétrico possuem valores similares com os parâmetros determinados com o experimento base, realizado com configuração densa de pontos de apoio planialtimétrico. A distância focal, determinada no experimento base, foi a que apresentou maior variabilidade (aproximadamente $0,012 \mathrm{~mm}$ ) em relação aos demais valores determinados. Os EMQ dos resíduos das coordenadas dos pontos de apoio nos quatro experimentos de calibração realizados encontram-se dentro das precisões admitidas no processo $(3 \mathrm{~cm})$. Considerando os EMQ dos resíduos das fotocoordenadas, provenientes dos processos de calibração, apresentados na tabela 02, verificam-se que todos os valores são inferiores ao valor de desvio padrão tolerável $(0,004 \mathrm{~mm})$. Esses valores 
permitem concluir que as medidas manuais dos pontos fotogramétricos realizadas no software LPS (Leica Photogrammetry Suite), encontram-se conforme as precisões admitidas no processo.

Em cada um dos experimentos realizados foi empregado um conjunto de pontos de apoio pré-sinalizados como sendo pontos de verificação para a análise da exatidão do processo (pontos sinalizados como círculos na figura 1). As coordenadas GPS desses pontos foram comparadas com as coordenadas geodésicas determinadas nos processos de calibração realizados. A Tabela 3 apresenta $o$ número de pontos de verificação utilizado e os EMQ das discrepâncias das coordenadas dos pontos de verificação nos quatro experimentos de calibração em serviço realizados com duas faixas de voo.

Tabela 3 - EMQ das discrepâncias das coordenadas dos pontos de verificação nos experimentos de calibração em serviço.

\begin{tabular}{ccccc}
\hline Experimento & $\begin{array}{c}\mathbf{n}^{\circ} \text { Pontos } \\
\text { de Verificação }\end{array}$ & $\begin{array}{c}\text { EMQ DX } \\
(\mathbf{m})\end{array}$ & $\begin{array}{c}\text { EMQ DY } \\
(\mathbf{m})\end{array}$ & $\begin{array}{c}\text { EMQ DZ } \\
(\mathbf{m})\end{array}$ \\
\hline 1 & 5 & 0,089 & 0,036 & 0,239 \\
2 & 5 & 0,393 & 0,408 & 0,289 \\
3 & 5 & 0,428 & 0,434 & 0,317 \\
4 & 5 & 0,538 & 0,508 & 0,724 \\
\hline
\end{tabular}

Valores toleráveis de $0,23 \mathrm{~m}$ e $0,64 \mathrm{~m}$ foram admitidos para a exatidão planimétrica e altimétrica de coordenadas tridimensionais determinadas na calibração via interseção fotogramétrica. Esses valores foram calculados com base na dimensão de um pixel na imagem, na altura de voo média, na aerobase média e no valor da distância focal da câmara (ALBERTZ e KREILING, 1980).

Verifica-se na Tabela 3 que somente o experimento 1 alcançou exatidões toleráveis nos pontos de verificação, pois os EMQ das discrepâncias das coordenadas planialtimétricas possuem valores menores que os valores toleráveis. Nos experimentos de calibração 2 e 3 , realizados com as configurações propostas de pontos de apoio altimétrico, somente a exatidão altimétrica foi alcançada, uma vez que os EMQ em altimetria foram inferiores ao valor admissível. Por outro lado, apesar dos valores de EMQ das discrepâncias planimétricas serem, aproximadamente, duas vezes maiores que valor tolerável, as exatidões obtidas nas calibrações podem ser aceitáveis se for considerada a utilização de uma câmara digital de baixo custo na execução de trabalhos de mapeamento fotogramétrico. Analisando-se os resultados obtidos nos experimentos realizados, verifica-se que a configuração com somente um ponto de apoio altimétrico foi a que apresentou as piores exatidões planimétricas e altimétrica na determinação tridimensional, conforme esperado.

$\mathrm{Na}$ tabela 4 são apresentadas as correlações estatísticas entre os POI e os POE determinados nos experimentos de calibração realizados. Neste trabalho adotou-se como sendo correlação alta o valor próximo ou superior a 0,7 (correlação de $70 \%$ ). 
Valores de correlação maiores ou iguais a essa tolerância são apresentados em negrito. Analisando a tabela 4 verifica-se a ocorrência de correlação entre os parâmetros $\omega$ com $y_{0}$ em todos os experimentos, sendo que no experimento que utiliza pontos de apoio planialtimétricos, esta correlação é a maior. A maior correlação entre os parâmetros de orientação das imagens com a posição do ponto principal, neste experimento, é justificada pela fixação das coordenadas planialtimétricas dos pontos de apoio no espaço objeto. A utilização de injunções nos pontos de apoio exigiu maiores adaptações nas posições e orientações das imagens para melhor adequação geométrica do espaço objeto. Como as coordenadas dos centros de projeções no espaço objeto também estão injuncionadas, a determinação da posição do ponto principal tornou-se mais dependente das compensações angulares exigidas para a fixação tridimensional dos raios de luz.

Tabela 4 - Correlação entre POI e POE nos experimentos de calibração em serviço com duas faixas de voo.

\begin{tabular}{|c|c|c|c|c|c|c|c|c|c|c|c|}
\hline \multicolumn{6}{|c|}{ Experimento 1 } & \multicolumn{6}{|c|}{ Experimento 2} \\
\hline & $\mathrm{f}$ & $\mathrm{x}_{0}$ & $\mathrm{~V}$ & $\mathrm{k}_{1}$ & $\mathrm{k}_{2}$ & & $\mathrm{f}$ & $\mathrm{x}_{0}$ & $\mathrm{y}_{0}$ & $\mathrm{k}_{1}$ & $\mathrm{k}_{2}$ \\
\hline$\omega$ & 0,046 & 0,117 & 0,896 & 0,018 & 0,034 & $\omega$ & 0,096 & 0,237 & 0,629 & 0,023 & 0,053 \\
\hline$\varphi$ & 0,034 & 0,534 & 0,420 & 0,022 & 0,016 & $\varphi$ & 0,341 & 0,348 & 0,313 & 0,030 & 0,027 \\
\hline$\kappa$ & 0,025 & 0,044 & 0,068 & 0,021 & 0,019 & $\kappa$ & 0,055 & 0,443 & 0,095 & 0,032 & 0,021 \\
\hline $\mathrm{X}_{0}$ & 0,013 & 0,073 & 0,014 & 0,013 & 0,015 & $\mathrm{X}_{0}$ & 0,038 & 0,060 & 0,013 & 0,011 & 0,013 \\
\hline $\mathrm{Y}_{0}$ & 0,006 & 0,021 & 0,027 & 0,007 & 0,008 & $\mathrm{Y}_{0}$ & 0,013 & 0,020 & 0,024 & 0,008 & 0,009 \\
\hline $\mathrm{Z}_{0}$ & 0,078 & 0,049 & 0,060 & 0,026 & 0,030 & $\mathrm{Z}_{0}$ & 0,112 & 0,096 & 0,044 & 0,025 & 0,027 \\
\hline \multicolumn{6}{|c|}{ Experimento 3} & \multicolumn{6}{|c|}{ Experimento 4} \\
\hline & $\mathrm{f}$ & $\mathrm{x}_{0}$ & - & $\mathrm{k}_{1}$ & $\mathrm{k}_{2}$ & & $\mathrm{f}$ & $\mathrm{x}_{0}$ & 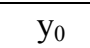 & $\mathrm{k}_{1}$ & $\mathrm{k}_{2}$ \\
\hline$\omega$ & 0,116 & 0,214 & 0,692 & 0,037 & 0,102 & $\omega$ & 0,114 & 0,213 & 0,691 & 0,035 & 0,099 \\
\hline$\varphi$ & 0,256 & 0,323 & 0,369 & 0,039 & 0,063 & $\varphi$ & 0,363 & 0,289 & 0,331 & 0,042 & 0,055 \\
\hline$\kappa$ & 0,036 & 0,443 & 0,063 & 0,024 & 0,022 & $\kappa$ & 0,068 & 0,439 & 0,065 & 0,024 & 0,022 \\
\hline $\mathrm{X}_{0}$ & 0,035 & 0,059 & 0,010 & 0,011 & 0,012 & $\mathrm{X}_{0}$ & 0,025 & 0,059 & 0,010 & 0,011 & 0,012 \\
\hline$Y_{0}$ & 0,011 & 0,019 & 0,019 & 0,008 & 0,009 & $\mathrm{Y}_{0}$ & 0,008 & 0,019 & 0,019 & 0,008 & 0,009 \\
\hline $\mathrm{Z}_{0}$ & 0,098 & 0,104 & 0,041 & 0,025 & 0,025 & $\mathrm{Z}_{0}$ & 0,090 & 0,103 & 0,039 & 0,026 & 0,025 \\
\hline
\end{tabular}

Os resultados das exatidões planialtimétricas obtidas no experimento 1 mostram que pequenas alterações nos parâmetros de orientação propiciaram melhores exatidões. $O$ que demonstra que essas exatidões estão fortemente relacionadas com a melhor adequação dos parâmetros de orientação das imagens para atender a injunção tridimensional no espaço objeto. Apesar da existência de correlações entre os parâmetros de orientação das imagens com a posição do ponto principal, os resultados obtidos nas calibrações realizadas possuem entre eles variações máximas de $0,004 \mathrm{~mm}$. Valor esse que pode ser assumido como sendo insignificante, pois encontra-se dentro da precisão de determinação no ajustamento MMQ realizado. Entretanto, os valores de exatidões obtidas nos experimentos são 
distintos. Resultado esse, que comprova a existência de forte compensação angular na orientação das imagens a fim de prover melhor adequação dos raios de luz no espaço objeto, uma vez que as orientações das imagens foram assumidas como sendo variáveis no ajustamento realizado. Este fato, também pode ser atribuído à fraca geometria do bloco (composto somente por duas faixas de voo) e, pelo pequeno número de pontos de apoio utilizado nestes experimentos.

Segundo Andrade (2003) é necessário ao menos uma foto girada em torno do eixo z da câmara em $90^{\circ}$ para separar os parâmetros de distorção radial simétrica daqueles da distorção descentrada. $\mathrm{O}$ uso de ao menos uma fotografia variando o ângulo Kappa em aproximadamente $90^{\circ}$ também atenua a correlação entre POI e POE (Merchant, 1980). Os experimentos apresentados na tabela 05 utilizaram de faixas de voo cruzadas, ou seja, variando o ângulo Kappa em aproximadamente $90^{\circ}$.

Tabela 5 - Experimentos de calibração em serviço com faixas cruzadas.

\begin{tabular}{ccc}
\hline Experimento & Configuração do Bloco & Parâmetros Injuncionados \\
\hline 5 & Faixas cruzadas & 24 Pontos de Apoio Planialtimétrico \\
6 & Faixas cruzadas & 24 Pontos de Apoio Altimétrico \\
7 & Faixas cruzadas & 5 Pontos de Apoio Altimétrico \\
8 & Faixas cruzadas & 1 Pontos de Apoio Altimétrico \\
\hline
\end{tabular}

No experimento 5 foram utilizados 24 pontos de apoio planialtimétrico homogeneamente distribuídos nas quatro faixas de voo (pontos apresentados como triângulos e quadrados na figura 1). Este experimento, assim como o experimento 1, serviu somente como referência aos experimentos de calibração em serviço com pontos de apoio altimétrico. O experimento 6 utilizou 24 pontos de apoio altimétrico homogeneamente distribuídos nas quatro faixas de voo (pontos apresentados como triângulos e quadrados na figura 1). No experimento 7 utilizou-se cinco pontos de apoio altimétrico, quatro nos extremos do bloco e 1 no centro (pontos sinalizados como quadrados na figura 1). $\mathrm{O}$ experimento 8 foi realizado com somente 1 ponto de apoio altimétrico no centro do bloco fotogramétrico (ponto sinalizado com um quadrado na figura 1).

Assim como nos experimentos com duas faixas, em todos os experimentos da tabela 5 a posição do $\mathrm{CP}$ da câmara foi utilizada como ponto de apoio (desvio padrão de $10 \mathrm{~cm}$ ). Foi empregado como desvio padrão das fotocoordenadas $1 / 2$ pixel $(0,004 \mathrm{~mm})$ e como desvio padrão das coordenadas de campo $3 \mathrm{~cm}$. A tabela 6 contém os valores dos POI significativos na análise da matriz variância e covariância do ajustamento, os seus desvios padrão, o EMQ dos resíduos das coordenadas dos pontos de apoio e centros de projeção e o EMQ dos resíduos das fotocoordenadas resultantes em cada um dos experimentos de calibração em serviço com faixas cruzadas. 
Tabela 6 - POI e EMQ das coordenadas dos Pontos de Apoio nos experimentos de calibração em serviço utilizando faixas de voo cruzadas.

\begin{tabular}{|c|c|c|c|c|}
\hline \multicolumn{5}{|c|}{ Parâmetros de Orientação Interior } \\
\hline & 5 & 6 & 7 & 8 \\
\hline $\mathrm{f}(\mathrm{mm})$ & 34,249 & 34,249 & 34,251 & 34,241 \\
\hline$\sigma_{\mathrm{f}}(\mathrm{mm})$ & 0,001 & 0,002 & 0,003 & 0,004 \\
\hline $\mathrm{x}_{0}(\mathrm{~mm})$ & 0,064 & 0,063 & 0,064 & 0,063 \\
\hline$\sigma_{\mathrm{x} 0}(\mathrm{~mm})$ & 0,001 & 0,001 & 0,001 & 0,001 \\
\hline $\mathrm{y}_{0}(\mathrm{~mm})$ & 0,272 & 0,273 & 0,274 & 0,276 \\
\hline$\sigma_{\mathrm{y} 0}(\mathrm{~mm})$ & 0,002 & 0,003 & 0,003 & 0,003 \\
\hline $\mathrm{k}_{1}\left(\mathrm{~mm}^{-2}\right)$ & $-9,120 \mathrm{E}-05$ & $-9,137 \mathrm{E}-05$ & $-9,134 \mathrm{E}-05$ & $-9,150 \mathrm{E}-05$ \\
\hline$\sigma_{\mathrm{k} 1}\left(\mathrm{~mm}^{-2}\right)$ & $3,678 \mathrm{E}-07$ & $3,688 \mathrm{E}-07$ & $3,732 \mathrm{E}-07$ & $3,608 \mathrm{E}-07$ \\
\hline $\mathrm{k}_{2}\left(\mathrm{~mm}^{-4}\right)$ & $1,043 \mathrm{E}-07$ & $1,048 \mathrm{E}-07$ & $1,045 \mathrm{E}-07$ & $1,048 \mathrm{E}-07$ \\
\hline$\sigma_{\mathrm{k} 2}\left(\mathrm{~mm}^{-4}\right)$ & $8,717 \mathrm{E}-10$ & $8,789 \mathrm{E}-10$ & $8,922 \mathrm{E}-10$ & $8,628 \mathrm{E}-10$ \\
\hline \multicolumn{5}{|c|}{ EMQ dos resíduos das coordenadas dos pontos de apoio e CPs } \\
\hline EMQ X (m) & 0,005 & - & - & - \\
\hline EMQ Y (m) & 0,006 & - & - & - \\
\hline EMQ Z (m) & 0,003 & 0,003 & 0,005 & 0,001 \\
\hline $\operatorname{EMQ~X}_{0}(\mathrm{~m})$ & 0,034 & 0,029 & 0,026 & 0,026 \\
\hline $\mathrm{EMQ} \mathrm{Y}_{0}(\mathrm{~m})$ & 0,036 & 0,035 & 0,034 & 0,033 \\
\hline EMQ $Z_{0}(\mathrm{~m})$ & 0,069 & 0,066 & 0,068 & 0,066 \\
\hline \multicolumn{5}{|c|}{ EMQ dos resíduos das fotocoordenadas } \\
\hline EMQ x (mm) & 0,001 & 0,001 & 0,001 & 0,001 \\
\hline EMQ y $(\mathrm{mm})$ & 0,002 & 0,002 & 0,002 & 0,002 \\
\hline
\end{tabular}

Considerando os EMQ dos resíduos das fotocoordenadas, provenientes dos processos de calibração, apresentados na tabela 06, verificam-se que todos os valores são inferiores ao valor de desvio padrão tolerável $(0,004 \mathrm{~mm})$. Os EMQ dos resíduos das coordenadas dos pontos de apoio de todos os experimentos de calibração, apresentados na tabela 6 , encontram-se dentro das precisões admitidas no processo $(3 \mathrm{~cm})$.

Comparando-se os resultados obtidos nas calibrações com o uso ou não de faixas cruzadas, verifica-se que as variações dos parâmetros de orientação interior, obtidas nas calibrações com o uso de pontos de apoio planialtimétrico, encontram-se próximas das suas precisões de determinação dos parâmetros. Entretanto, quando pontos de apoio altimétrico são empregados, observa-se uma variação significativa entre as distâncias focais e posições do ponto principal determinadas. Essas diferenças estão relacionadas com maior definição geométrica ao processo de calibração com a utilização de faixas cruzadas. Assim, os parâmetros determinados nos experimentos com pontos de apoio altimétrico e faixas cruzadas aproximam-se dos determinados nos experimentos de referência (1 e 5). Por outro lado, o comportamento de distorção radial simétrica, como pode ser visto na Figura 2, possui comportamento praticamente igual em todas as calibrações realizadas. 
Figura 02: Curvas de distorção radial simétrica das oito calibrações realizadas.

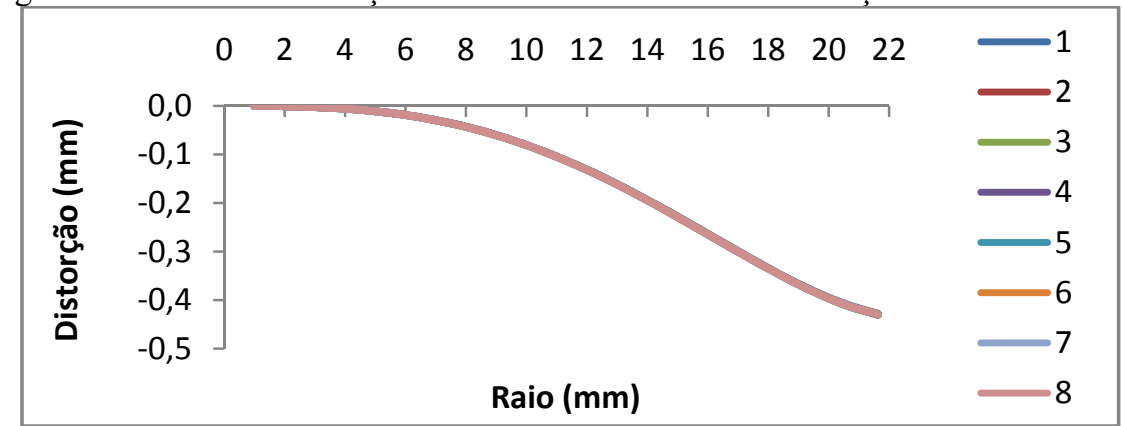

Igualmente como empregado nos experimentos de calibração sem o uso de faixas cruzadas, pontos pré-sinalizados que não participaram do processo de calibração foram utilizados como pontos de verificação no processo (pontos sinalizados com círculos na figura 1). A Tabela 7 apresenta o número de pontos de verificação e os EMQ das discrepâncias de coordenadas dos pontos de verificação nos 4 experimentos de calibração com faixas cruzadas.

Analisando-se os valores de EMQ das discrepâncias das coordenadas dos pontos de verificação, obtidos nos processos de calibração apresentados na tabela 7 , verifica-se que todos possuem valores inferiores ou muito próximos às tolerâncias planimétrica e altimétrica adotadas, exceto o experimento 8. Pode-se observar que houve uma maior exatidão na reconstrução do espaço objeto quando comparado com os resultados obtidos na calibração em serviço sem o uso de faixas de voo cruzadas e com as configurações de pontos de apoio altimétrico.

Tabela 7 - EMQ dos resíduos das coordenadas dos pontos de verificação nos experimentos de calibração em serviço com faixas cruzadas.

\begin{tabular}{ccccc}
\hline Experimento & $\begin{array}{c}\mathbf{n}^{\circ} \text { Pontos } \\
\text { de Verificação }\end{array}$ & $\begin{array}{c}\text { EMQ X } \\
(\mathbf{m})\end{array}$ & $\begin{array}{c}\text { EMQ Y } \\
(\mathbf{m})\end{array}$ & $\begin{array}{c}\text { EMQ Z } \\
(\mathbf{m})\end{array}$ \\
\hline 5 & 9 & 0,097 & 0,036 & 0,105 \\
6 & 9 & 0,289 & 0,181 & 0,158 \\
7 & 9 & 0,281 & 0,173 & 0,171 \\
8 & 9 & 0,425 & 0,247 & 0,509 \\
\hline
\end{tabular}

Os experimentos 1 e 5, realizados para fins de referência nas análises dos resultados obtidos nas calibrações com o uso de pontos de apoio altimétrico, não apresentam variações significativas, tanto nos POI como também nos resultados das exatidões planialtimétricas obtidas nos pontos de verificação. Por outro lado, as calibrações realizadas com pontos de apoio altimétrico e faixas cruzadas, apesar da pequena variação nos POI, aumentaram consideravelmente a exatidão planialtimétrica da reconstrução do espaço objeto. Mesmo com a configuração de 
somente um ponto de apoio altimétrico no centro do bloco, os resultados são melhores do que os obtidos sem o uso de faixas cruzadas, apesar de ainda apresentar precisão planimétrica inferior ao valor tolerável. Percebe-se claramente que os POI obtidos nos experimentos 5 a 8 são mais estáveis se comparados com os obtidos nos experimentos de 1 a 4 e ainda, os POI resultantes do experimento 1 são os que mais se aproximam dos POI resultantes dos experimentos 5 a 8 .

Para complementar as análises realizadas dos resultados obtidos com a calibração com o uso de faixas cruzadas e pontos de apoio altimétrico, na tabela 8 são apresentadas as correlações estatísticas entre os POI e os POE determinados nos experimentos de calibração realizados.

Analisando-se a tabela 08 constata-se que a utilização de faixas cruzadas acrescentou maior rigidez geométrica ao processo, o que fez com que as correlações existentes entre os parâmetros de orientação das imagens e de posição do ponto principal, existentes nos experimentos anteriores, fossem aqui atenuadas. E ainda, assegurou que os parâmetros de orientação das imagens fossem determinados com exatidões necessárias para permitir a interseção tridimensional dentro dos padrões de tolerâncias adotadas. A simples comparação entre os POI da tabela 2 com os da tabela 6 revela que pequenas alterações nesses parâmetros foram suficientes para aumentar as exatidões planialtimétricas na reconstrução do espaço objeto. Os resultados obtidos nos experimentos mostraram que a calibração em serviço com a utilização de pontos de apoio altimétrico e as coordenadas das estações de exposição requer o uso de faixas de voo cruzadas para aumentar a rigidez geométrica do bloco, assegurando uma melhor determinação dos parâmetros de orientação interior e exterior.

Tabela 8 - Correlação entre POI e POE nos experimentos de calibração em serviço com faixas de voo cruzadas.

\begin{tabular}{|c|c|c|c|c|c|c|c|c|c|c|c|}
\hline \multicolumn{6}{|c|}{ Experimento 5} & \multicolumn{6}{|c|}{ Experimento 6} \\
\hline & $\mathrm{f}$ & $\mathrm{x}_{0}$ & $\mathrm{y}_{0}$ & $\mathrm{k}_{1}$ & $\mathrm{k}_{2}$ & & $f$ & $\mathrm{x}_{0}$ & $y_{0}$ & $\mathrm{k}_{1}$ & $\mathrm{k}_{2}$ \\
\hline$\omega$ & 0,018 & 0,289 & 0 & 0,027 & 0,044 & $\omega$ & 0,213 & & & 0,039 & 0,054 \\
\hline$\varphi$ & 0,020 & 0,2 & 0 & 0,029 & 0,045 & $\varphi$ & 0,215 & & & 0,026 & 0,047 \\
\hline$\kappa$ & 0,016 & 0 , & & 0,013 & 0,0 & & 54 & & & 0 , & 0,023 \\
\hline $\mathrm{X}_{0}$ & 0,006 & 0 , & 0 , & 0,008 & 0,009 & $\mathrm{X}_{0}$ & 19 & 0, & 5 & 0,007 & 0,008 \\
\hline $\mathrm{Y}_{0}$ & 0,006 & 0,032 & 0, & 0,007 & 0,008 & $\mathrm{Y}_{0}$ & 0,0 & 0,0 & 0 , & 0,006 & 0,007 \\
\hline$Z_{0}$ & 0,059 & 0,035 & 0,034 & 0,022 & 0,026 & $\mathrm{Z}_{0}$ & 83 & 0,065 & 0,031 & 0,022 & 0,025 \\
\hline \multicolumn{6}{|c|}{ Experimento 7} & \multicolumn{6}{|c|}{ Experimento 8} \\
\hline & $\mathrm{f}$ & $\mathrm{x}_{0}$ & $\mathrm{y}_{0}$ & $\mathrm{k}_{1}$ & $\mathrm{k}_{2}$ & & $\mathrm{f}$ & $\mathrm{x}_{0}$ & & $\mathrm{k}_{1}$ & $\mathrm{k}_{2}$ \\
\hline$\omega$ & 0,170 & 0,269 & 0,421 & 0,035 & 0,052 & $\omega$ & 0,239 & 0,255 & 0,411 & 0,027 & 0,047 \\
\hline$\varphi$ & 0,182 & 0,266 & 0,418 & 0,022 & 0,047 & $\varphi$ & 0,257 & 0,249 & 0,402 & 0,025 & 0,049 \\
\hline$\kappa$ & 0,062 & 0,366 & 0,023 & 0,022 & 0,020 & $\kappa$ & 0,063 & 0,366 & 0,031 & 0,018 & 0,017 \\
\hline $\mathrm{X}_{0}$ & 0,017 & 0,030 & 0,015 & 0,007 & 0,008 & $\mathrm{X}_{0}$ & 0,014 & 0,030 & 0,015 & 0,007 & 0,008 \\
\hline $\mathrm{Y}_{0}$ & 0,013 & 0,027 & 0,016 & 0,006 & 0,007 & $\mathrm{Y}_{0}$ & 0,010 & 0,028 & 0,016 & 0,006 & 0,006 \\
\hline$Z_{0}$ & 0,069 & 0,069 & 0,032 & 0,022 & 0,024 & $\mathrm{Z}_{0}$ & 0,072 & 0,068 & 0,033 & 0,021 & 0,022 \\
\hline
\end{tabular}




\section{CONCLUSÕES}

O objetivo principal deste artigo foi de avaliar o uso da posição do $\mathrm{CP}$ da câmara e diferentes configurações de pontos de apoio altimétrico na calibração em serviço de uma câmara digital de baixo custo. Concentrou-se ainda, na análise da correlação entre POI e POE quando se utiliza um recobrimento aéreo convencional e um recobrimento com o uso de faixas cruzadas na calibração.

Os procedimentos de calibração de câmaras em serviço, utilizando a posição do CP da câmara e pontos de apoio altimétrico, são viáveis, mas requerem do uso de faixas de voo cruzadas para se alcançar à exatidão planimétrica adotada nesta pesquisa, como também para minimizar as correlações entre os parâmetros de orientação das imagens e coordenadas do ponto principal. Os procedimentos de calibração, com a utilização de recobrimento aéreo convencional e apoio altimétrico, alcançaram exatidão planimétrica igual a dois pixels no terreno; o que pode ser considerado como sendo aceitável na execução de trabalhos de mapeamento fotogramétrico com a utilização de uma câmara digital de baixo custo. No caso de se utilizar faixas de voo convencionais torna-se necessário o emprego de um número maior de pontos de apoio para ser alcançada a exatidão planimétrica requerida.

As calibrações em serviço, empregando pontos de apoio planialtimétrico foram as que apresentaram as melhores exatidões planimétricas, independente da utilização de faixas cruzadas. Por outro lado, quando somente as duas faixas de voo com recobrimento convencional foram utilizadas, correlações entre parâmetros de orientação das imagens e as coordenadas do ponto principal foram acentuadas. Entretanto, com a utilização de faixas cruzadas, as correlações entre parâmetros com efeitos similares foram atenuadas. Dessa forma, se for considerado um processo de calibração que visa somente à determinação de POI, conclui-se que a utilização de faixas cruzadas é condição imprescindível para qualquer um dos procedimentos de calibração empregados nessa pesquisa.

A calibração em serviço, com o uso pontos de apoio altimétrico nos extremos e no centro do bloco e as coordenadas das estações de exposição, alcançou as precisões planimétrica e altimétrica esperadas nesta pesquisa quando faixas cruzadas foram adicionadas ao bloco.

Trabalhos futuros concentrarão na calibração em serviço com base em informações altimétricas advindas da nuvem de pontos LIDAR e na verificação do desempenho dos POI, determinados nas calibrações em serviço, para a realização de aerotriangulações de blocos fotogramétricos (empregando as coordenadas das estações de exposição), obtidos em locais e épocas diferentes dos que foram empregados nos procedimentos de calibração.

\section{AGRADECIMENTOS:}

Os autores agradecem ao Lactec - Instituto de Tecnologia pela disponibilização das fotografias aéreas e dos dados LIDAR e a CAPES 
(Coordenação de Aperfeiçoamento de Pessoal de Nível Superior) pela disponibilização de bolsa de estudos durante parte desta pesquisa.

\section{REFERÊNCIAS BIBLIOGRÁFICAS}

ALBERTZ, J., KREILING, W. Photogrammetric Guide. Ed. Wichmann, Karlsruhe, 1980.

ANDRADE, J.B. Fotogrametria. Curitiba, UFPR, 2003.

BAZAN, W.S., TOMMASELLI, A.M.G., GALO, M., RUY, R.S. Calibração de um sistema dual de câmaras digitais convergentes. II Simpósio Brasileiro de Geomática V Colóquio Brasileiro de Ciências Geodésicas. Presidente Prudente, 2007.

BROWN, D. C., 1971. Close-range camera calibration. Ibid., 37(8): 855-866.

CLARKE, T. A., FRYER, J. G. The development of camera calibration methods and models Photogrammetric Record, v. 16, nº1, p. 51-66, abril, 1998.

CÔRTES, J. B. R. Análise da estabilidade geométrica de câmaras digitais de baixo custo com diferentes métodos de calibração. Tese (Doutorado em Ciências Geodésicas) Universidade Federal do Paraná, Curitiba, Paraná, 2010.

CRONK, S., FRASER, C., HANLEY, H. Automated calibration of colour digital cameras. Photogrammetric Record, v. 21 n$^{\circ} 116$, p. 355-372, dezembro, 2006.

GALO, M., TOMMASELLI, A. M. G. Calibração de Câmaras. In PITERI, M. A., RODRIGUES, J. C. (Ed.). Fundamentos de visão computacional. Presidente Prudente, SP: FCT/UNESP-PP, 2011.

HABIB, A. F., MORGAN, M., LEE, Y., Bundle Adjustment with self calibration using straight lines. Photogrammetric Record, v. 17 n¹00, p. 635-650, outubro, 2002.

HABIB, A., MORGAN, M. Automatic calibration of low cost digital cameras. Journal of Optical Engineering, v. $42 \mathrm{n}^{\circ} 4$ p. 948-955, Abril 2003.

HABIB, A. F., PULLIVELLI, M., MORGAN, M. Quantitative measures for the evaluation of camera stability. XX International Symposium Photogrammetry and Remote Sensing, Istanbul, Turquia, TS WG I/2 Sensor Calibration and Testing, p.63, Julho, 2004.

HABIB, A., PUlliVElli, A., MITISHITA, E., GHANMA, M., KIM, E.M. Stability analysis of low-cost digital cameras for aerial mapping using different georeferencing techniques. The Photogrammetric Record. v.21, n¹13 p. 2943, 2006.

HABIB, A., JARVIS, A., DETCHEV, G., STENSAAS, D., MOE, D., CHRISTOPHERSON, J. Standards and specifications for the calibration and stability of amateur digital camaras for close-range mapping applications. International Archives of Photogrammetry, Remote Sensing and Spatial Information Sciences, vol XXXVII parte B1, Beijin, 2008.

HONKAVAARA, E. Calibrating digital photogrammetric airbone imaging systems using a test field. Tese (Doutorado em Ciência de Tecnologia), Helsinki University of Technology, Finlândia, 2008. 
HONKAVAARA, E., et al. A permanent test field for digital photogrammetric systems. Photogrammetric Engineering \& Remote Sensing v. 74 n 1 , p. 95 106, janeiro, 2008.

LERMA, J.L., CABRELLES, M. A review and analyses of plumb-line calibration. Photogrammetric Record. v. 22 n¹18, p.135-150, junho, 2007.

MARTINS, M. Geração de ortoimagem a partir de georreferenciamento direto de imagens digitais aérea de pequeno formato com dados lidar. Dissertação (Mestrado em Ciências Geodésicas) Universidade Federal do Paraná, Curitiba, Paraná, 2010.

MERCHANT, D.C. Analytical Photogrammetry: Theory and Practice. The Ohio State University, 1980.

MITISHITA, E., CÔRTES, J., CENTENO, J., MACHADO, A. M.L. Small-format digital camera: a study into stability analysis of the interior orientation parameters through temperature variation. MMT09 $6^{\text {th }}$ International Symposium on Mobile Mapping Technology, Presidente Prudente, Brasil, 2009.

MitiShitA, E., CÔRTES, J., CENTENO, J., MACHADO, A.M.L., MARTINS, M. Study of stability analysis of the interior orientation parameters from the small-format digital camera using on-the-job calibration. Canadian Geomatics Conference. Calgary, Alberta, 2010.

REMONDINO, F. FRASER, C. Digital camera calibration methods considerations and comparisons. In: International Archives of Photogrammetry and Remote Sensing, v. XXXVI, parte 5, 2006.

RUY, R.S., TOMASELLI, A.M.G., GALO, M., HASEGAWA J.K., MENOSSI, R.C. Fototriangulação com parâmetros adicionais para câmaras digitais: uma avaliação experimental. Boletim de Ciências Geodésicas. v. 14 n4, p 571-587, out-dez, 2008.

WEGMANN, H. HEIPKE, C., JACOBSEN, K. Direct sensor orientation based on GPS network solutions. International Archives of Photogrammetry and Remote Sensing, XXXV, p 153-158, Istanbul, 2004.

YASTIKLI, N., JACOBSEN K., Influence of system calibration on direct sensor orientation. Photogrammetric Engineering and Remote Sensing. v. 5 p. 629633, maio, 2005.

YASTIKLI, N. TOTH, C., BRZEZINSKA. In-situ camera and boresight calibration with LIDAR data. The $5^{\text {th }}$ International Symposium on mobile mapping technology. Padua, Itália, 2007.

(Recebido em outubro de 2011. Aceito em janeiro de 2012.) 